\title{
Hepatological aspects of Epstein-Barr virus infection in children
}

\author{
Justyna Moppert ${ }^{1}$, Małgorzata Pawłowska ${ }^{1,2}$ \\ 'The Tadeusz Browicz Provincial Hospital for Infectious Diseases and Observation, Bydgoszcz, Poland \\ 2Department of Infectious Diseases and Hepatology, Ludwik Rydygier Collegium Medicum in Bydgoszcz, \\ Nicolaus Copernicus University in Torun, Bydgoszcz, Poland
}

\section{ABSTRACT}

The Epstein-Barr virus (EBV), belonging to the Herpesviridae family, is widespread in the world today. The most typical features of infectious mononucleosis include a triad of symptoms in the form of fever, cervical lymphadenopathy, and pharyngitis. Primary infection in the youngest children is usually asymptomatic and self-limiting. Hepatitis affects a significant proportion of patients and is usually a mild and self-limiting condition. Cholangitis is less common in the paediatric population and requires only symptomatic treatment in most cases. In contrast, in patients with immunodeficiency, EBV infection may be the cause of acute multiple organ failure and death. EBV infection plays an important and multifaceted role in the liver and biliary tract pathology due to its widespread distribution in the paediatric population.

KEY WORDS:

cholecystitis, hepatitis, infectious mononucleosis.

\section{INTRODUCTION}

Epstein-Barr virus (EBV) infections are widespread throughout the world. They affect over $95 \%$ of adults, while in developed countries about $50 \%$ of children up to five years of age are found to have antibodies that indicate this infection [1]. EBV infections in children are usually asymptomatic. Infection in adolescents and adults can lead to the development of infectious mononucleosis, most often with a triad of symptoms being fever, cervical lymphadenopathy, and pharyngitis $[2,3]$. Primary EBV infection mainly affects children and adolescents, while patients in immunosuppression most often reactivate the latent form of infection [4]. In the acute phase of primary EBV infection, an increase in liver enzymes is often observed whereas a mild to moderate increase in transaminases affects 80 $90 \%$ of patients. Severe cholestatic hepatitis occurs in only
$5 \%$ of cases in patients with infectious mononucleosis [5, 6]. Hepatic failure or pancreatitis complicating cholestatic hepatitis are extremely rare in the course of EBV infection [6-9]. The mechanism by which hepatitis-related pancreatitis develops in the course of EBV infection is unknown and probably multifactorial. An unusual symptom of infectious mononucleosis is also acute cholecystitis, isolated thickening of the gallbladder wall, or cholicele.

Gilbert's syndrome may also play an important role in the development of gallbladder abnormalities in patients with cholestatic hepatitis in the course of primary EBV infection $[5,9,10]$.

\section{HEPATITIS AND HEPATIC FAILURE}

A mild increase in transaminases (up to about 3 times above the upper limit of normal - ULN) is observed in

\section{ADDRESS FOR CORRESPONDENCE:}

Justyna Moppert, The Tadeusz Browicz Provincial Hospital for Infectious Diseases and Observation, 12 św. Floriana St., 85-030 Bydgoszcz, Poland, ORCID: 0000-0001-6299-2103, e-mail: j_slagowska83@o2.pl 
about $90 \%$ of patients infected with EBV and is mainly asymptomatic [2, 5, 7, 11-13]. Transaminases exceeding 10 -fold ULN occur significantly less frequently and require the extension of hepatological diagnostics $[11,13$, 14]. Systematic increases in alanine aminotransferase (ALT) and aspartate aminotransferase (AST) are most often observed during the second week of the disease, while normalisation is observed in the next 2-6 weeks [13]. Kofteridis et al. observed an increase in liver enzymes as early as in the first week of the disease, with maximum activity in the second week and a tendency to normalise from the third week [11]. Splenomegaly or hepatomegaly are common abnormalities on abdominal ultrasound and affect approximately $25 \%$ and $12 \%$ of children with infectious mononucleosis, respectively [14].

Severe cholestatic hepatitis characterised by increased alkaline phosphatase (ALP), gamma-glutamyl transpeptidase (GGTP), or hyperbilirubinaemia are rarely seen in the acute phase of EBV infection and affect approximately $5-6.6 \%$ of patients $[5,6,11-14]$. Clinically overt yellowing of the integuments may be associated not only with cholestasis but also with haemolytic anaemia resulting from infection with the $\operatorname{EBV}[5,13]$.

The mechanism for the development of EBV-induced hepatitis is not clear; the EBV does not directly destroy liver cells or the bile duct epithelium. Studies show that T CD8+ lymphocytes infected by the virus accumulate in the liver and activate pro-inflammatory cytokines such as interferon $\gamma$ or tumour necrosis factor $\alpha$ (TNF- $\alpha$ ), which directly damage hepatocytes $[6,14]$.

Severe liver damage in the form of fulminant hepatitis failure (FHF) is extremely rare. These cases mainly concern patients with immunodeficiency, e.g. HIV-infected, after organ transplants, or patients with X-linked lymphoproliferative syndrome (Duncan syndrome). This syndrome was described for the first time by Purtilo et al. and concerned only boys [12-16].

In early childhood, $75 \%$ of children with Duncan syndrome develop fulminant hepatic failure in the course of primary acute EBV infection, which is most often the cause of their death. Reye's syndrome can also be the cause of fulminant hepatitis with hyperammonaemia and encephalopathy. The syndrome was first described by Reye in 1963. Most often it develops during the recovery period after a viral infection during which acetylsalicylic acid was used. In addition to the EBV, possible infectious agents inducing Reye's syndrome include adenoviruses, influenza, parainfluenza, and Coxsackie viruses [17].

Acute liver failure may also be a complication of EBV-dependent lymphoproliferative syndrome developing after organ transplantation (post-transplant lymphoproliferative disorder - EBV-PTLD). In recent years, it has been proven that in approximately $90 \%$ of cases, EBV infection is responsible for the occurrence of PTLD in children. This is associated with a higher percentage of EBV-seronegative people in younger age groups, which is an important predictor when receiving an organ from an EBV-seropositive donor. Apart from age and serological status, another risk factor for the development of PTLD is the type of organ transplant and the associated intensity of immunosuppression $[4,18]$.

Feranchak et al. [16] published a description of the first case of liver transplantation in a child with fulminant hepatitis (FHF) in the course of primary EBV infection. It concerned a 22-month-old, immunocompetent girl with unencumbered perinatal and family history, in whom the disease began with febrile states, vomiting, and diarrhoea during the two days preceding hospitalisation, and yellowing of the integuments 24 hours before admission to hospital. Apart from twice-taken acetaminophen $(13 \mathrm{mg} / \mathrm{kg})$, the child did not receive any other medicines. Serum acetaminophen concentration was within the therapeutic range. Based on the features of coagulopathy (prothrombin time $23.5 \mathrm{~s}$ and $33.4 \mathrm{~s}$ in the first and second days of hospitalisation, respectively), high transaminases, histopathological examination results obtained during liver biopsy, and detection of EBV DNA viraemia, FHF induced by an EBV infection was diagnosed. After liver transplantation, the patient underwent immunosuppressive and anti-viral treatment. Up to two years after transplantation, the child had no clinical evidence of EBV infection, immune dysfunction, or lymphoproliferative disease. The publication also reviews the literature on FHF in children in the course of acute infectious mononucleosis from 1949 to 1998 . The report describes nine patients aged between 12 weeks and 17 years, among which only in one case was there no death. Nakazawa et al. in 2015 described five patients with FHF induced by an EBV infection among 44 paediatric patients hospitalised in 2006-2014 to qualify them for liver transplantation. None of these children had any previous history of immune, metabolic, or hepatological disorders [19].

In immunocompetent persons, the course of acute EBV infection is most often asymptomatic. Latent EBV remains in B lymphocytes and may contribute to recurrent clinical symptoms resulting from periodic reactivation of the infection. Chronic active EBV (CAEBV) is an example of a chronic mononucleosome-like infection with the presence of a high titre of antibodies against EBV antigens, which suggests active replication. This clinical form of chronic EBV infection was originally described in children, but in recent years, along with the development of diagnostic methods, it has also been increasingly reported in adults [20]. Chronic active EBV infection is characterised by recurrent fever, lymphadenopathy, hepatosplenomegaly, and increased transaminases and is complicated by high mortality.

The relationship between EBV infection and the development of autoimmune diseases still requires further research. In adult patients, both acute infection and reactivation of primary EBV infection are considered possible predictors of the development of autoimmune diseases 
including AIH. In children, this effect is less obvious and there are few reports on it [21]. Nobili et al. showed a possible relationship between EBV infection and the development of type $1 \mathrm{AIH}$ in a five-year-old child, while the potential impact of EBV infection in a child on the development of AIH-2 was described in 2013 by Zellos et al. [21, 22].

In children, especially with prolonged cholestatic hepatitis and results of serological tests confirming EBV infection, autoimmunological hepatitis should be considered in the differential diagnosis, especially if anti-LKM 1 autoantibodies are detected in the serum [21]. In such cases, a liver biopsy should be performed to establish a final diagnosis.

\section{ACUTE ACALCULOUS CHOLECYSTITIS}

Acute acalculous cholecystitis (AAC) is defined as cholecystitis without deposits. In paediatric patients it accounts for $50-70 \%$ of all cases of acute cholecystitis. In adults this percentage is much lower and amounts to $5-10 \%[5,23,24]$. The criteria confirming the diagnosis of AAC in an ultrasound study are thickening of the gallbladder wall above $3 \mathrm{~mm}$ or dilatation of the gallbladder lumen, the presence of pericholecystic fluid, and condensed bile in the lumen. An additional clinical symptom confirming the diagnosis is pain located at Murphy's point. The combination of a minimum of two ultrasound criteria and clinical symptoms are sufficient to make a diagnosis $[5,25,26]$.

In the paediatric population, $\mathrm{AAC}$ is most commonly observed in the course of viral infections, e.g. EBV, HAV, HHV-6; however, some AAC cases also occur in the course of non-infectious disorders such as long-term parenteral nutrition, Kawasaki disease, extensive burns, or surgical procedures [24,25]. Poddighe et al. reviewed the literature on the occurrence of AAC in children in the years 2000-2018. In 14 cases, the effect of EBV infection on the development of acalculous cholecystitis was confirmed. No patient underwent cholecystectomy. Among children undergoing antibiotic therapy, third-generation cephalosporin or ciprofloxacin and metronidazole were used [24]. The main treatment recommended in patients with AAC following primary EBV infection is still fluid therapy, analgesic, and nutritional treatment while broad-spectrum antibiotics are used in cases of secondary bacterial superinfection [9].

The mechanisms supporting the development of AAC are not clear. Possible impaired blood supply or hypotension of the gallbladder and bile condensation with or without bile duct obstruction are assumed [23, 24]. Gilbert's syndrome should also be included in the pathogenesis of EBV-induced acalculous cholecystitis in the paediatric population because it occurs in 11-16\% of Caucasian individuals and is a hereditary and mild hyperbilirubinaemia caused by a mutation in the UGT1A1 gene encoding the UDP-glucuronyl transferase (UDPG) enzyme $[1,5,9,10]$. In about $40 \%$ of cases, the disease is caused by a mutation consisting of the insertion of the TA dinucleotide in the promoter region of the UGT1A1 gene. The mutation leads to impaired bilirubin conjugation in hepatocytes. Guala et al., based on a study of patients hospitalised due to prolonged fever in the course of infectious mononucleosis, showed that infection with the EBV can lead to liver function damage, especially in homozygous TA7/TA7 or heterozygous TA6/TA7 individuals for the UDPGT1 promoter gene [26].

The case for a correlation between EBV infection, acute acalculous cholecystitis, and Gianotti-Crosti syndrome (GCS) in a child was first described Wu and Huang in 2013 [27]. This is a rare disease, most often occurring in children between 3 months and 15 years of age, with the peak incidence between 1 and 6 years of age, and sometimes seen in adults. The correlation of EBV infection and GCS is not uncommon, while this relationship in combination with the occurrence of AAC is extremely rare [27].

In the paediatric population, EBV infection has a very broad symptomatology, ranging from an asymptomatic course to fulminant hepatitis with severe cholestasis, which is why it is so important to know the possible hepatological complications associated with EBV infection, which in rare cases may be a condition threatening the overall health and even the life of the child.

\section{DISCLOSURE}

The authors declare no conflict of interest.

\section{REFERENCES}

1. Colombini E, Guzzo I, Morolli F, at al. Viral load of EBV DNAemia is a predictor of EBV-related post-transplant lymphoproliferative disorders in pediatric renal transplant recipients. Pediatr Nephrol 2017; 32: 1433-1442.

2. Arya S, Saini A, El-Baba M, at al. Epstein-Barr virus-associated acute acalculous cholecystitis: a rare occurrence but favorable outcome. Clin Pediatr (Phila) 2010; 49: 799-804.

3. Cohen JI. Epstein-Barr Virus Infection. N Engl J Med 2000; 343: 481-492.

4. Styczyński J, Gil L, Kyrcz-Krzemień S, et al. Strategy of management in Epstein-Barr virus infections in hematology, oncology and transplantology. Guidelines of Polish Federation of Bone Marrow Transplant Centers. Acta Haematologica Polonica 2012; 43: 48-53.

5. Attilakos A., Prassouli A, Hadjigeorgiou G, et al. Acute acalculous cholecystitis in children with Epstein-Barr virus infection: a role for Gilbert's syndrome? Int J Infect Dis 2009; 13: 161-164.

6. Shkalim-Zemer V, Shahar-Nissan K, Ashkenazi-Hoffung L, at al. Cholestatic Hepatitis Induced by Epstein-Barr Virus in a Pediatric Population. Clin Pediatr 2015; 54: 1153-1157.

7. Negro F. The paradox of Epstein-Barr virus-associated hepatitis. J Hepat 2006; 44: 839-841.

8. Kang SJ, Yoon KH, Hwang JB. Epstein-Barr virus infection with acute pancreatits associated with cholestatic hepatitis. Pediatr Gastroenterol Hepatol Nutr 2013; 16: 61-64. 
9. Kottanattu L, Lava S, Helbing R, et al. Pancreatitis and cholecystitis in primary acute symptomatic Epstein-Barr virus infection - Systematic review of literature. J Clin Virol 2016; 82: 51-55.

10. Kobayashi HA, Hong KW, Lee JS, at al. A case of acute cholecystitis without cholestiasis caused by Epstein-Barr virus in a healthy young woman. Int J Infect Dis 2016; 90: 330-335.

11. Kofteridis D, Koulentaki, Valachis A, at al. Epstein-Barr Virus hepatitis. Eur J Intern Med 2011; 22: 73-76.

12. Kimura H, Nagasaka T, Hoshino Y, at al. Severe hepatitis caused by Epstein-Barr virus without infection of hepatocyte. Hum Pathol 2001; 32: 757-762.

13. Crum N. Epstein-Barr Virus Hepatitis: Case Series and Review. South Med J 2006; 99: 544-547.

14. Yang SI, Geong JH, Kim JY. Clinical characteristics of Primary Epstein-Barr Virus Hepatitis with elevation of Alkaline Phosphatase and $\gamma$-Glutamylotransferase in Children. Yonsei Med J 2014; 55 : 107-112.

15. Gugig R, Rosenthal P. Fulminant hepatic failure in children. Therapy 2008; 5: 451-463.

16. Feranchak A, Tyson R, Narkewicz M, et al. Fulminant Epstein-Barr Viral hepatitis: Orthotopic Liver Transplantation and review of the Literature. Liver Transpl Surg 1998; 4: 469-476.

17. Chapman J, Arnold J. Reye Syndrome. StatPearls [Internet] Last Update: January 17, 2019; https://www.ncbi.nlm.nih.gov/books/ NBK526101/

18. Holmes R, Sokol R. Epstein-Barr virus and post-transplant lymphoproliferative Disease. Pediatr Transplant 2002: 6: 456-464.

19. Nakazawa A, Nakano N, Fukuda A, et al. Use of serial assessment of disease severity and liver biopsy for indication for liver transplantation in pediatric Epstein-Barr virus induced fulminant hepatic failure. Liver Transpl 2015; 21: 362-368.

20. Kimura H, Cohen J. Chronic active Epstein-Barr virus disease. Front Immunol 2017; 8: 1867.

21. Zellos A, Spoulou V, Roma-Giannikou E, et al. Autoimmune hepatitis type-2 and Epstein-Barr virus infection in a toddler: art of facts or an artifact? Ann Hepatol 2013; 12: 147-151.

22. Nobili V, Comparcola D, Sartorelli M, et al. Autoimmune hepatitis type I after Epstein-Barr virus infection. Pediatr Infect Dis J 2003; 22: 387.

23. Alkhoury F, Diaz D, Hidalgob J. Acute acalculous cholecystitis in the pediatric population associated with Epstein-Barr Virus Infection. Case report review of the literature. Int J Surg Case Rep 2015; 11: 50-52.

24. Poddighe D, Sazonov V. Acute acalculous cholecystitis in children. World J Gastroenterol 2018; 24: 4870-4879.

25. Imamoğlu M, Sarihan H, Sari A, Ahmetoğlu. Acute acalculous cholecystitis in children. Diagnosis and treatment. J Pediatr Surg 2002; 37: 36-39.

26. Guala A, Campra D, Marinelli I, et al. Are Gilbert's syndrome and liver involvement genetically linked in infectious mononucleosis? Pediatr Infect Dis J 2003; 22: 1110-1111.

27. Wu WJ, Huang DT. Primary Epstein-Barr virus associated acute acalculous cholecystitis and Gianotti-Crosti syndrome. Tzu Chi Med J 2013; 25: 51-54. 